\title{
Transição demográfica, acumulação de capital e progresso tecnológico: desafios para o crescimento brasileiro
}

\author{
João Basilio Pereima* \\ Alexandre Porsse ${ }^{* * *}$
}

\begin{abstract}
Resumo: Este artigo aborda os efeitos da transição demográfica sobre a capacidade de crescimento das economias e discute o caso da transição demográfica brasileira e destaca os efeitos restritivos que o fim do bônus demográfico e aumento da razão de dependência dos idosos irá impor sobre a taxa de investimento e poupança e, portanto, sobre o crescimento e compara a transição brasileira com o que ocorre no resto do mundo. $\mathrm{O}$ artigo faz ainda uma estimativa não paramétrica, usando método Kernel Epanechnikov para demonstrar as relações não lineares entre razão de dependência de idosos com crescimento do PIB, taxa de investimento e taxa de poupança para uma amostra de 140 países e conclui que a partir de uma razão de dependência de idosos de 8 a taxa de crescimento começa a cair e a partir de uma razão de $12 \%$ as taxas de investimento e poupança também caem. $\mathrm{O}$ artigo conclui que o caso brasileiro já está muito próximo do ponto de inflexão.
\end{abstract}

Palavras-chave: Crescimento, Demografia; Acumulação de Capital; Progresso Tecnológico.

Classificação JEL: 040; J10.

\footnotetext{
"Doutor em Desenvolvimento Econômico pela Universidade Federal do Paraná (UFPR). Professor adjunto do Departamento de Economia da Universidade Federal do Paraná (UFPR). Endereço eletrônico: joaobasilio@ufpr.br

** Doutor em Economia pela Universidade Federal do Rio Grande do Sul (UFRGS). Professor adjunto da Uiversidade Federal do Paraná (UFPR). Endereço eletrônico: joaobasilio@ufpr.br
} 


\section{Introdução}

A relação entre crescimento econômico e demografia tem sido reconhecida desde longa data. A mudança demográfica pode afetar o crescimento econômico por diversos mecanismos. As mudanças na estrutura da população têm impactos nas decisões de consumo e de poupança das famílias, na oferta de trabalho e produtividade, nos investimentos públicos já que os gastos do sistema de pensões tendem a aumentar, e por fim, impacta na capacidade da economia inovar a partir de um estoque crescente ou decrescente/estagnado de capital humano conforme o estágio demográfico em que o país se encontra. Em geral se reconhece que o aumento relativo da população em idade ativa (15 a 65) em relação à população total promove o crescimento ao passo que o aumento de populações jovens ( 0 a 15 ) e idosas tem impactos negativos (65 ou mais). No entanto pode haver casos especiais em que o aumento da idade da população pode ter efeito positivo, quando o aumento da idade média for seguido de aumento produtividade e salário. Neste caso o efeito demanda decorrente do repasse da produtividade aos salários mais do compensaria efeitos negativos do envelhecimento sobre o lado da oferta, ao reduzir a probabilidade ou a taxa de invenção de novos produtos e, portanto, de novos ciclos de expansão. As relações entre mudança demográfica e crescimento, pelos diversos canais pelos quais afeta a acumulação de capital e o progresso tecnológico estão longe de se caracterizar como processos simples e unívoco.

As intensas transformações econômicas e demográficas observadas ao longo do século XX inspiraram um considerável volume de estudos analisando teórica e empiricamente os efeitos de alterações demográficas sobre a trajetória de crescimento e bem estar de uma economia (Lee, 2003; Fukuda, 2004; Dyson, 2010; Weber, 2010; Shoven, 2011, entre inúmeros outros). Mais recentemente eventos demográficos diversos e marcantes ocorridos ao longo do século XX despertaram uma nova onda de estudos, teóricos e principalmente empíricos, analisando as relações complexas entre crescimento econômico e mudança demográfica. Muitos estudos reconhecem que a taxa de crescimento da renda e da população influenciam uma à outra de modo que ambas podem ser consideradas variáveis endógenas para efeitos de teorias de crescimento (Galor, 2011). Além destes trabalhos teóricos, inúmeros estudos de casos tem sido elaborados com o intuito de gerar compreensão de processos específicos de cada economia os quais geralmente são usados para subsidiar políticas públicas específicas que lidam com os impactos das mudanças demográficas. Entre estes estudos encontram-se casos como dos EUA (The National Academy of Sciences, (2012), Europa (Neyer et all, 2013); Ásia (Eggleton), Japão (Fukuda e Morozumi, 2004). Mudanças na estrutura demográfica no Brasil tem sido estudada por Carvalho (1997) e os impactos sobre regionais por Azzoni, Menezes-Filho e Menezes (2005) e Stampe et all, (2012). Enquanto que no Brasil os estudos tem destacado os efeitos positivos da passagem do bônus demográfico ao longo da década de 1970 até a década de 2000 , outros estudos em economias com regimes demográficos diferente, em especial com populações idosas, destacam os desafios e dificuldades 
em se manter o crescimento econômico e até mesmo a capacidade competitiva da economia via inovações. O caso japonês, conforme Fukuda e Morozumi (2004), é particularmente elucidativo, por se constituir na população média mais idosa entre os países industrializados, onde é possível observar os diversos efeitos que o envelhecimento populacional exerce sobre a atividade econômica.

Em geral a literatura teórica afirma que o crescimento econômico é impulsionado pelo chamado bônus demográfico, quando a população em idade de trabalho (15 a 65 anos) cresce em proporção à população jovem (0 a 15) e idosa (65 ou mais). A oferta crescente de mão obra no mercado de trabalho contribui para manter os salários relativamente baixos, embora possam aumentar, e ao mesmo tempo expandir a demanda por meio do aumento do estoque de trabalhadores, o que favorece ao fim o crescimento econômico, especialmente quando sustentado por consumo interno. Além deste mecanismo uma população mais jovem estaria numa fase do seu ciclo de vida em que estaria acumulando capital e, portanto, poupando à uma taxa mais alta, como forma de manter estável o padrão de vida quando atingir a idade de aposentadoria. Estes dois mecanismos, oferta de mão de obra e maior propensão a poupar são mecanismos já descritos e amplamente reconhecidos pela literatura (Horioka, 1991; Higgins and Willianson, 1996; Bloom and Willianson, 1998; e Bloom et all, 1999).

Muitos dos efeitos da transição demográfica sobre o crescimento e bem estar, embora previstos teoricamente, não são completamente conhecidos empiricamente. A importância de aprofundar estudos empíricos e até mesmo teóricos que revelem novas correlações e causalidades é enorme, especialmente num momento em que praticamente todos países passam por significativas mudanças demográficas, com quedas expressivas nas taxas de natalidade e mortalidade. A importância de aprofundar os estudos demográficos e econômicos no Brasil é maior ainda, na medida em que os trabalhos mais escassos e na medida em que o país apresenta um processo de transição demográfica numa velocidade maior do que a média mundial. Neste sentido nas próximas seções deste artigo apresentamos um panorama mundial da transição demográfica onde destacamos o Brasil como um processo de transição mais rápido que a economia mundial em termos da razão de dependência da população jovem, em idade ativa e idosa. Em seguida, na seção 2, apresentamos uma análise estatística não paramétrica, através da estimativa de uma função de densidade pelo método Kernel Epanechnikov para o caso univariado em uma amostra de 140 países por meio da qual demonstra-se relações não lineares entre crescimento, investimento e poupança e razão de dependência de idosos e adicionalmente, que o ponto de inflexão quando os efeitos passam a ser negativos ocorrem em torno de uma razão de dependência de idosos de 8 a $12 \%$ da população. Considerando que o Brasil está com uma relação de dependência de idosos de 7\%, muito próximo ao ponto de inflexão, na seção 3 do artigo analisamos as consequências de tal fato para o crescimento da economia brasileira nos próximos anos. $\mathrm{O}$ artigo conclui afirmando que há um espaço reduzido e que se esgota rapidamente para mais um ciclo de acumulação de capital e que doravante o crescimento da economia brasileira dependerá predominantemente de um processo baseado em inovação 
tecnológica sujeito as restrições do envelhecimento populacional. Em outras palavras, a era dos milagres de crescimento está acabando, se já não acabou para o Brasil.

\section{Panorama da transição demográfica no mundo e no Brasil}

Após um período de explosão demográfica deflagrada no final do século XVII, a humanidade culmina no início século XXI com o final de uma acelerada fase de transição em direção ao crescimento populacional zero, o qual já foi alcançado em alguns países enquanto que outros ainda vivem o período de desaceleração das taxas de crescimento populacional. A taxa de crescimento populacional tem caído sistematicamente ao longo da segunda metade do século $\mathrm{XX}$ em todas as regiões do planeta, embora ainda persistam diferentes níveis de taxas de crescimento populacional, onde países menos desenvolvidos crescem a taxas maiores que os países desenvolvidos, mas todas em declínio (gráfico 1).

Durante a primeira metade do século XX a maioria dos países desenvolvidos, especialmente europeus, experimentaram baixas taxas de fertilidade e crescimento populacional em parte causada pelas guerras. $\mathrm{O}$ período que antecede a primeira guerra mundial também foi marcado por baixo crescimento populacional e baixo crescimento da renda (gráfico 2a). Esse período coincide com baixas taxas de crescimento econômico, um período que suscitou teses de crescimento estagnacionistas. A segunda metade do século XX, por sua vez, é marcada por uma inflexão na taxa de fertilidade, especialmente nos países à época industrializados, como uma reação aos episódios dramáticos das duas guerras. Esta resposta demográfica coincidiu com a retomada do crescimento econômico materializado no que se convencionou chamar de "idade de ouro do capitalismo", que durou de 1950 a 1970 aproximadamente e que teve no fenômeno do "baby-boom" americano seu mais eloquente exemplo. Como pode ser observado no gráfico 1, a taxa de crescimento populacional foi alta em todas as regiões do planeta entre 1950-1970, comparado aos períodos mais recentes. Após os anos 1970 a taxa ingressou numa trajetória de queda sistemática e disseminada em todas as regiões a qual continuará em curso de redução ao longo de todo século XXI, segundo estimativas da Divisão de População das Nações Unidas. 
Gráfico 1 - Taxa de crescimento da população mundial 1950-2010 $3,00 \%$

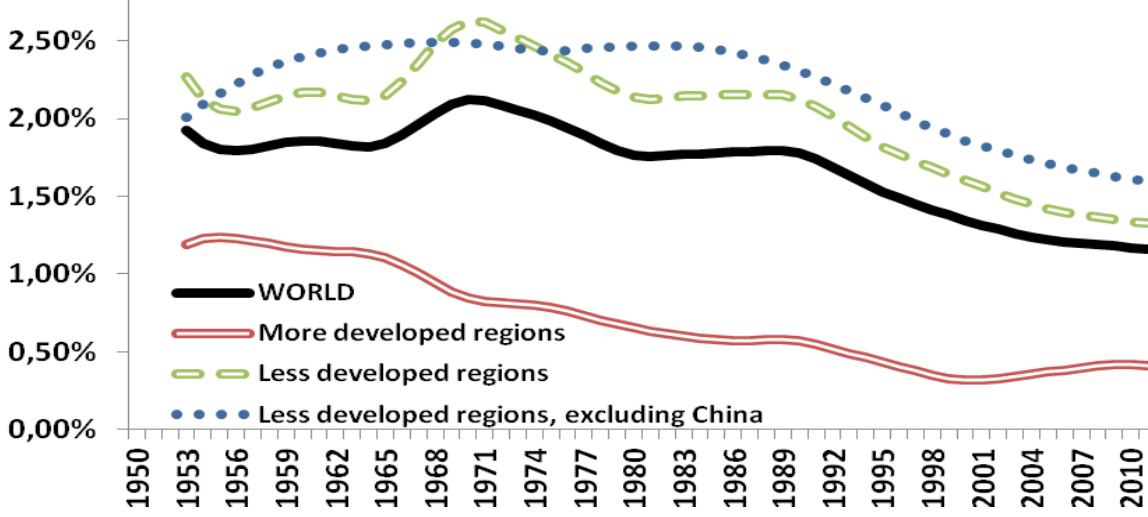

Fonte: ONU, Population Division. (Estimated Sheet).

A concomitância entre a elevada taxa de crescimento população e elevada taxa de crescimento da renda no período da idade de ouro do capitalismo (1950-1970) não é uma coincidência histórica. Vários estudos tem demostrado uma correlação positiva entre ambas. A forte correlação entre crescimento populacional e crescimento econômico está demonstrada no gráfico $2 \mathrm{~b}$, onde ajustamos uma reta que indica que um crescimento da ordem de $1 \%$ na população implica em crescimento de $1 \%$ no produto (0,92\% para ser mais exato). Os dados populacionais estão agregados e efeitos diferenciados de faixas de idade sobre o crescimento não são mostrados. Por exemplo, como demonstrado em Macunovich (2012), queda na taxa de crescimento populacional de faixas etárias específicas tem efeitos diferenciados sobre o crescimento da renda per-capita podendo ser negativo ou positivo.

Gráfico 2 - Crescimento anual PNB e população

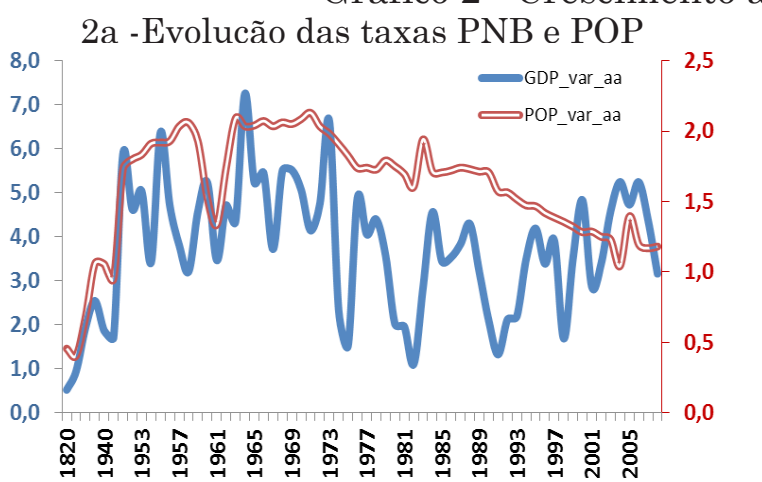

2b - Relacão entre taxas PNB e POP

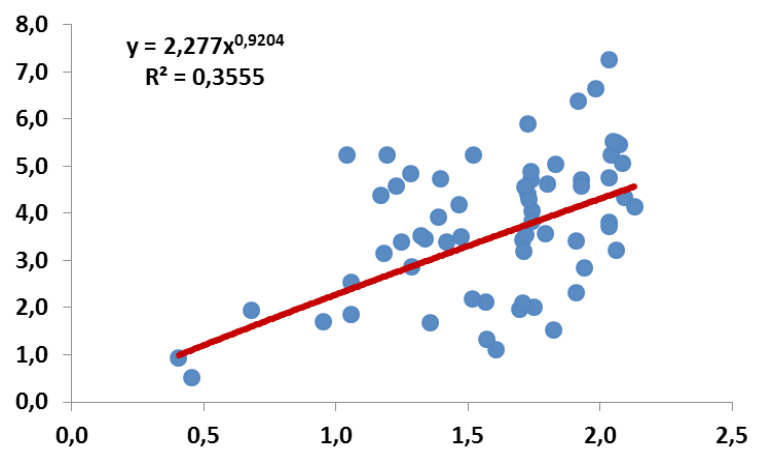

Fonte: Madison (2006), World Bank, United Nations-Population Division.

O caso da transição demográfica acelerada da economia brasileira já foi reconhecido na literatura por alguns estudos, dentre os quais destacam-se Carvalho (1997) e Berquó, Garcia e Lago (2008) e mais recentemente reconhecido por Arbache (2011), onde o autor explora as consequências para o mercado de trabalho e efeitos negativos que poderá ter sobre a produtividade. A fim de melhor evidenciar a transição demográfica brasileira com o resto do mundo, 
mostramos através dos gráficos 3a, 3b e 3c a evolução da razão de dependência da população infantil/jovem (0 a 15 anos), em idosa (65 anos ou mais) e em idade ativa (entre 15 e 64 anos). Os gráficos destacam a evolução da população brasileira (linha pontilhada) comparando-a com a média mundial tomada de uma amostra de 232 países (linha dupla). Como pode ser observado tanto a queda da razão de dependência da população jovem quanto o aumento da razão de dependência da população idosa são mais acelerados que a média mundial. No caso da razão de dependência de idosos (3b) o nível apresentava-se entre os mais baixos do mundo até o fim da década de 1980 e passou a aumentar mais intensamente dai em diante. A razão de dependência atingiu $10 \% \mathrm{em}$ 2010. Projeções do IBGE e ONU, conforme gráfico 4, mostram que ela atingirá 11,8\% em 2015 e 13,8\% em 2020. Como veremos na próxima seção esta razão de dependência é um ponto de inflexão que dará início à um regime de crescimento econômico mais baixo devido a diversas restrições demográficas.

Gráfico 3 - Evolução da razão de dependência no mundo e no Brasil
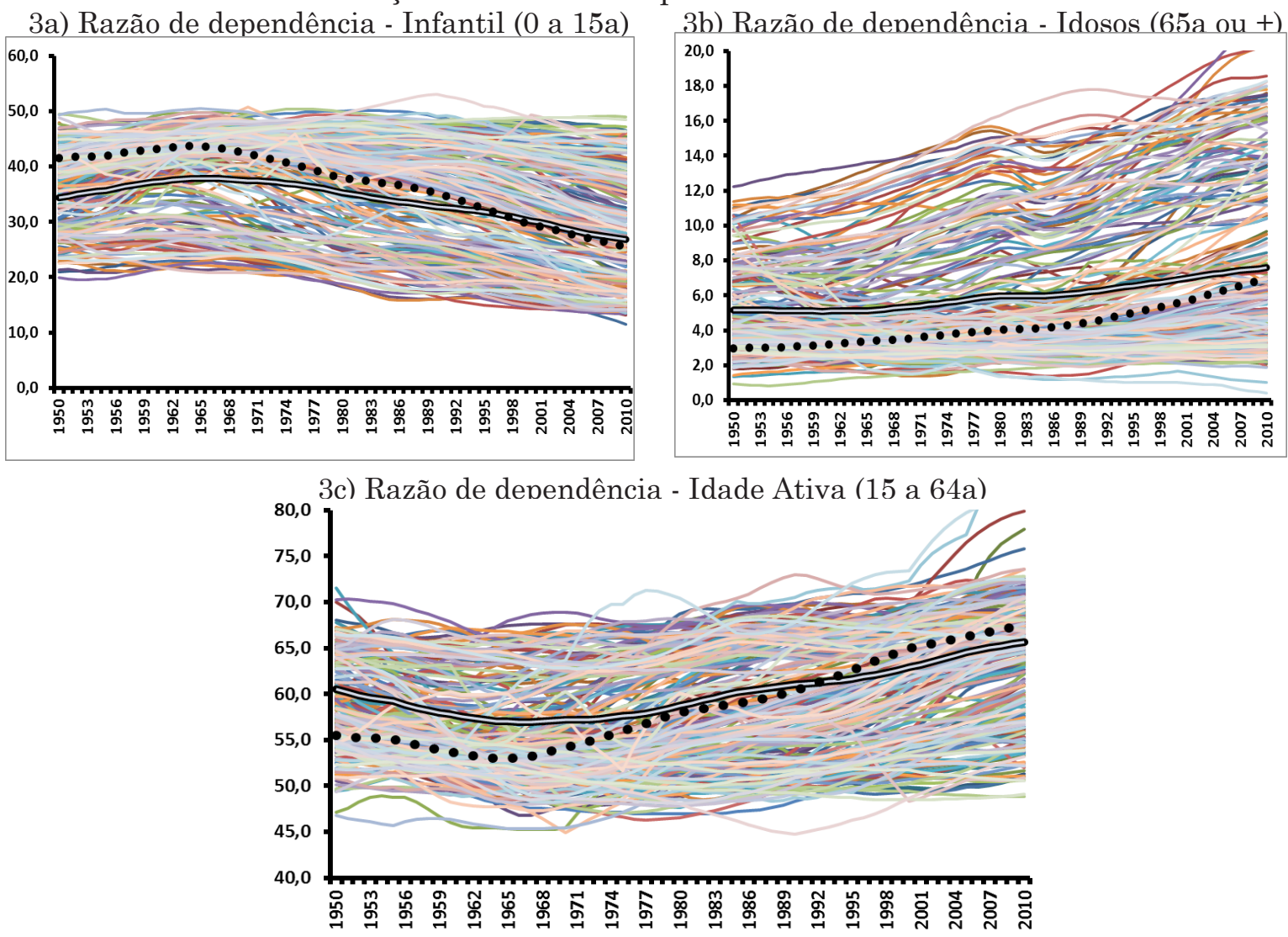

Fonte: ONU, Population Division. (Estimated Sheet).

Ainda segundo projeções do IBGE e ONU em 2030 a proporção de população brasileira jovem diminuirá devido à menor taxa de natalidade e parte significativa da população será concentrada entre 25 e 50 anos de idade, e por fim em 2050, boa parte da população envelhecerá e terá idade superior a 45 anos. Estas mudanças globais incorporam outros efeitos que merecem ser destacados, 
em especial os impactos sobre o bônus demográfico e sobre o crescimento da população economicamente ativa (PEA), a qual também pode ser interpretada como a oferta potencial de mão de obra da economia, desde que se assuma que todos desejem trabalhar. Os próximos dois gráficos mostram estas relações. No gráfico 4, pode-se observar que o crescimento da população (linha vermelha) atingirá o máximo em 2040, quando o total alcançar 224 milhões de habitantes. No entanto as projeções da PEA, a população entre 15 e 64 anos, indicam que ela atingirá o máximo e começará a cair com uma década de antecedência. $\mathrm{E}$ mais ainda, conforme mostrado no gráfico 5 mais adiante, o bônus demográfico terminará com duas décadas de antecedência em relação à população total. Em ambos os casos os impactos econômicos da transição demográfica no Brasil acontecerão mais cedo do que em geral tem-se assumido. Acreditamos que estas duas antecipações ainda não estão muito bem avaliadas pela sociedade brasileira, muito embora nos meios acadêmicos o assunto já tenha sido muito estudado por Arbache (2011), Alvez et all (2010) e Mason (2005), entre outros. A queda antecipada da PEA e o fim do bônus demográfico é de grande interesse, pois a primeira afeta diretamente o mercado de trabalho enquanto o segundo atinge a estrutura da demanda efetiva, com impactos na composição da cesta de consumo da sociedade, na taxa de poupança e na capacidade de crescimento da economia.

O bônus demográfico já dura 50 anos desde que começou em 1960, no entanto sua passagem está com os dias contados. Terminará em 2020, daqui 8 anos, ou mais dois mandatos presidenciais apenas. O bônus demográfico pode ser visto com clareza no gráfico 4, onde mostramos a evolução da razão de dependência da população brasileira. O significado da razão de dependência é intuitivo e mostra o percentual da população improdutiva ou dependente, aquela que pelo motivo idade não trabalha e, portanto, não produz, em relação a população total. Uma razão de dependência de $60 \%$, por exemplo, significa que $60 \%$ da população (jovens e idosos) dependem da população economicamente ativa, aquela situada entre 15-64 anos de idade, que será de 40\% do total.

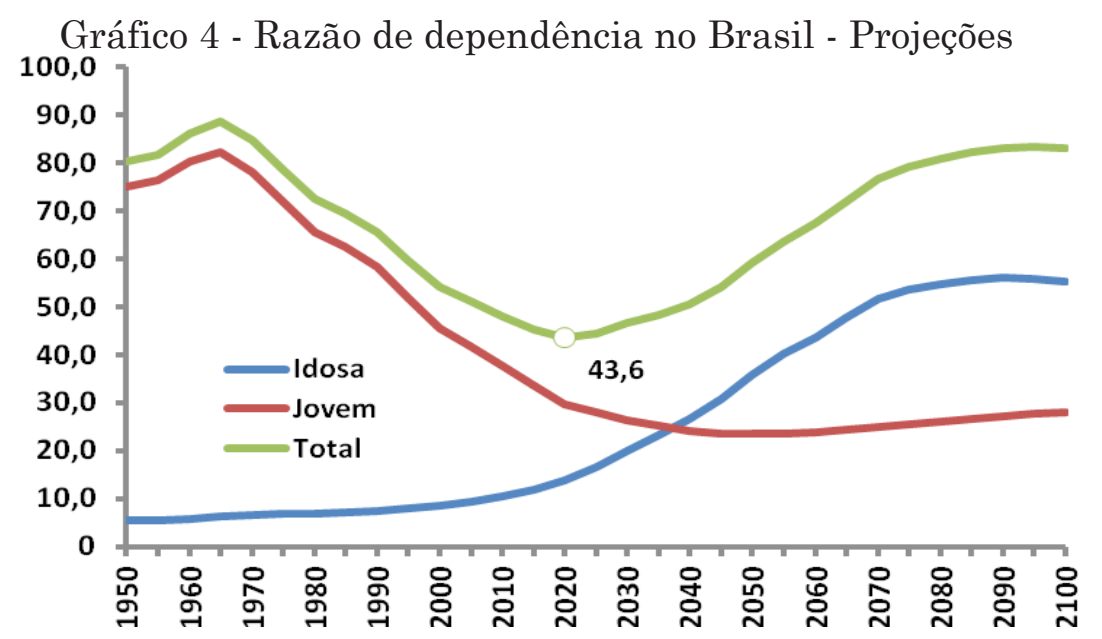

Fonte: IBGE, United Nations, Department of Economic and Social Affairs: Population Division, Population Estimates and Projections Section: http://esa.un.org/wpp/Excel-Data/population.htm. Mudanças 
demográficas pós 2010: projeções com base no cenário médio.

Gráfico 5 - População total e 15-64 anos (PEA)

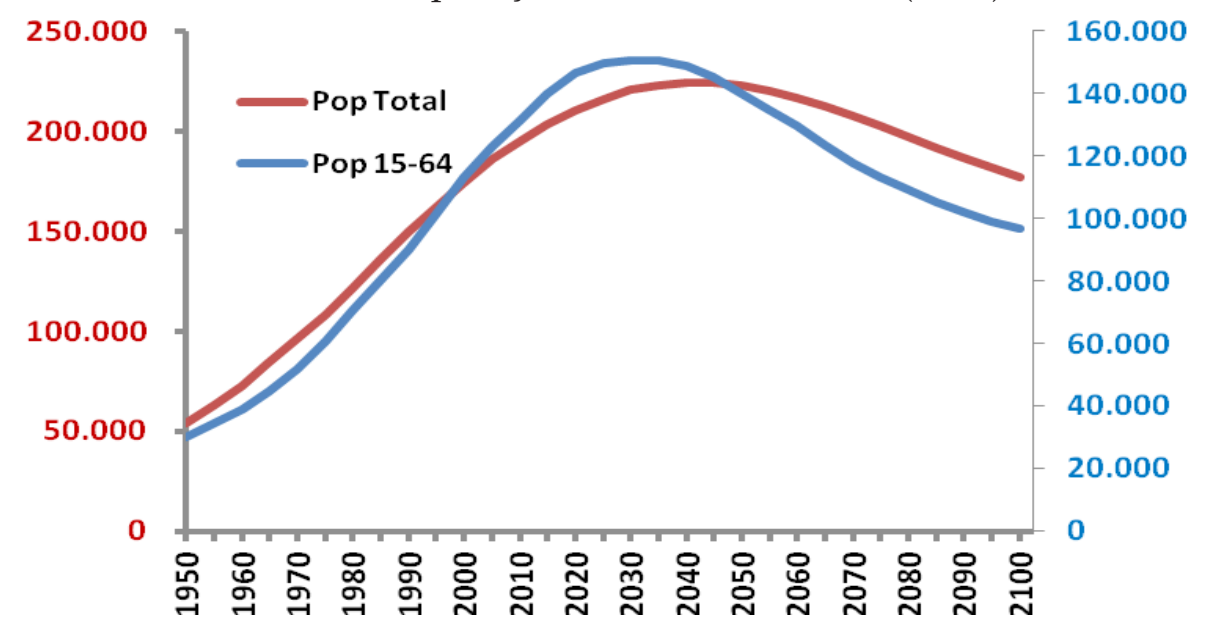

Fonte: IBGE, United Nations, Department of Economic and Social Affairs: Population Division, Population Estimates and Projections Section: http://esa.un.org/wpp/Excel-Data/population.htm. Mudanças demográficas pós 2010: projeções com base no cenário médio.

\section{Transição demográfica, acumulação de capital e progresso tecnológico}

A literatura sobre crescimento econômico e transição demográfica tem demonstrado que há uma forte correlação entre estrutura etária de uma população, na forma da razão de dependência e taxa de crescimento. Os primeiros modelos de crescimento de diferentes vieses, como Harrod(1939) e Domar(1946), Solow(1956) e Swan(1956), Cass (1965) e Koopmans (1965), tratam a variável demográfica de forma agregada assumindo uma taxa exógena de crescimento da força de trabalho sem considerações maiores sobre sua composição. Em geral as conclusões a que chegaram estes modelos é de que no longo prazo e na ausência de progresso tecnológico, a taxa de crescimento de equilíbrio será igual ao crescimento da força de trabalho. Neste ponto de equilíbrio o aprofundamento do capital (Domar 1946) chegou a tal ponto que já absorveu toda a mão de obra disponível, de forma que doravante a relação capital-trabalho $(K / L)$, capitalproduto $(K / Y)$ e produto-trabalho $(Y / L)$ serão constante. Todas as variáveis no chamado stady-state estão crescendo à mesma velocidade de forma a manter a relação entre elas constante. Neste caso a renda em nível estará crescendo, mas a taxa de crescimento da renda per capita será zero, a menos que se varie a taxa de poupança (acumulação de capital) ou a produtividade (progresso tecnológico ou inovação). É de particular interesse para este trabalho destacar que nestas teorias que o período de aceleração do crescimento coincide com um período de abundância de mão-de-obra em relação ao capital de tal forma uma elevada taxa de poupança pode se converter em acumulação de capital fazendo aumentar a relação capital-trabalho. Isto ocorrerá até que a exaustão da oferta de mão de obra. A queda na taxa de crescimento da força de trabalho pode antecipar a chegada deste momento. E de fato, como visto na seção anterior, 
a desaceleração das taxas de crescimento populacional e o envelhecimento da população na maioria dos países está conduzindo a economia mundial para um novo regimente de crescimento em que diminuirá o crescimento econômico baseado em acumulação de capital. A continuidade do crescimento da renda per capita dependerá então da capacidade de cada economia em obter aumentos de produtividade e aumento na taxa de invenção de novos produtos. Para tal é necessário que o fim de um regime de crescimento econômico baseado em acumulação de capital coincida com um período de acumulação de capital humano de forma a preparar o mercado de trabalho e a economia como um todo para um regime de crescimento baseado em inovação.

A relação entre crescimento econômico agregado, acumulação de capital medida pela taxa de investimento ou formação brita de capital fixo e taxa de poupança pode ser constatada por meio da estimação de um função de densidade pelo método Kernel - Epanechnikov, para cada uma das variáveis: crescimento do produto nacional bruto (GDP ou PIB), formação bruta de capital fixo ou taxa de investimento como proporção do produto (FBC), e taxa de poupança (POUP). Como o método Kernel calcula a inclinação de uma curta reta ajustada dentro de um intervalo de observações próximos, a sucessão de retas conectadas ao longo de todos os intervalos de observações agrupados permite extrair uma curva estimada de forma não paramétrica, isto é, sem impor-lhes uma forma funcional pré-definida, seja ela linear, exponencial, polinômica ou qualquer outra forma arbitrária.

O que os Kernels indicam, de acordo com o gráfico 6, é que de fato, existe uma relação não linear entre razão de dependência de idosos (RDI) e PIB, FBC e POUP. Está relação é positiva para baixos valores de razão de dependência e negativa para altos. Além disto, um resultado importante do Kernel é que ele mostra também qual é valor da razão de dependência em que esta inversão ocorre. Este valor é muito parecido para as três variáveis, PIB, FBC e POUP e situa-se em torno de $8 \%$ para o crescimento do PIB e $12 \%$ para FBC e POUP. $\mathrm{O}$ que estes resultados permitem concluir, do ponto de vista de regime de crescimento, é que o crescimento econômico baseado em acumulação de capital começa se esgotar quando a razão de dependência de idosos ultrapassa a marca dos $8 \%$. 
Gráfico 6 - Crescimento, acumulação de capital, poupança e transição demográfica

Kerne I Fit (Epanechnikov, $\mathrm{h}=\mathbf{2 . 5 7 1 8}$ )

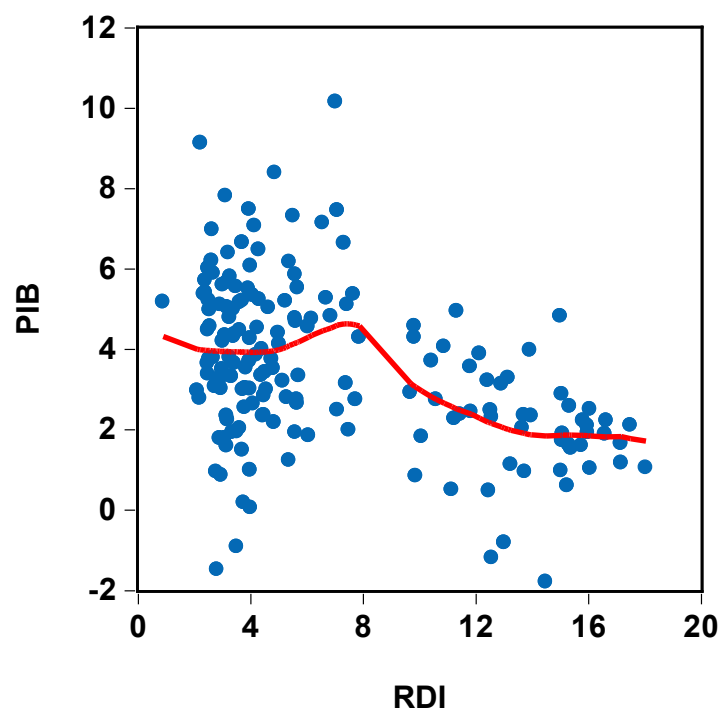

Kernel Fit (Epanechnikov, $\mathrm{h}=\mathbf{2 . 5 7 1 8 )}$

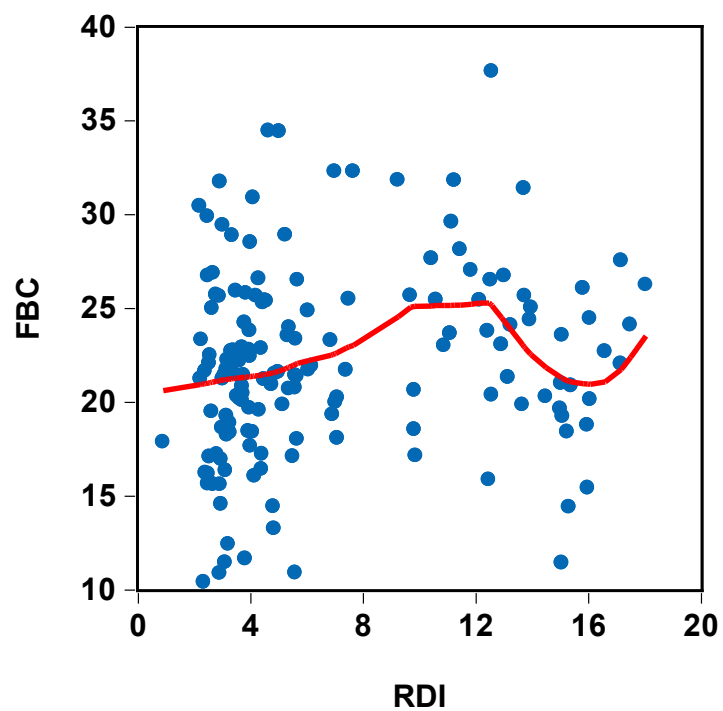

Kernel Fit (Epanechnikov, $h=2.5718)$

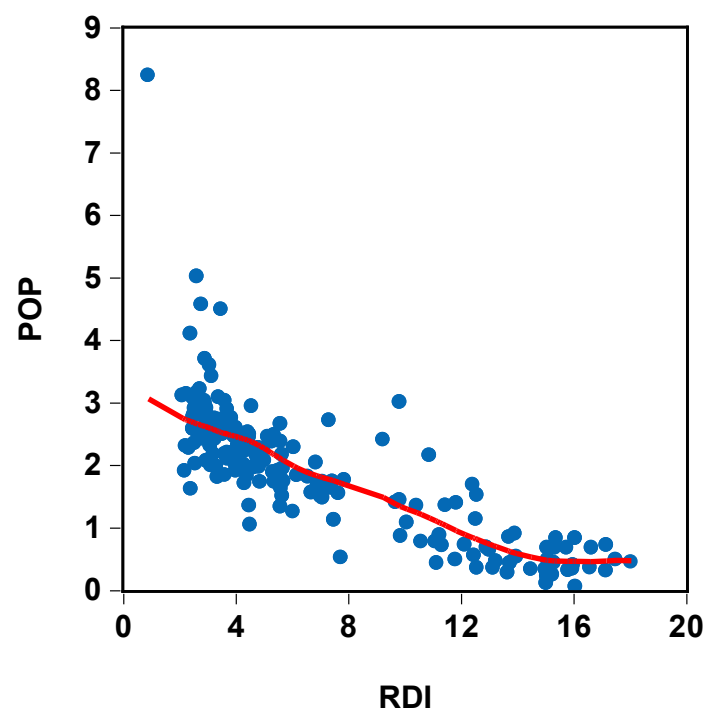

Kernel Fit (Epanechnikov, $\mathrm{h}=\mathbf{2 . 5 7 1 8 )}$

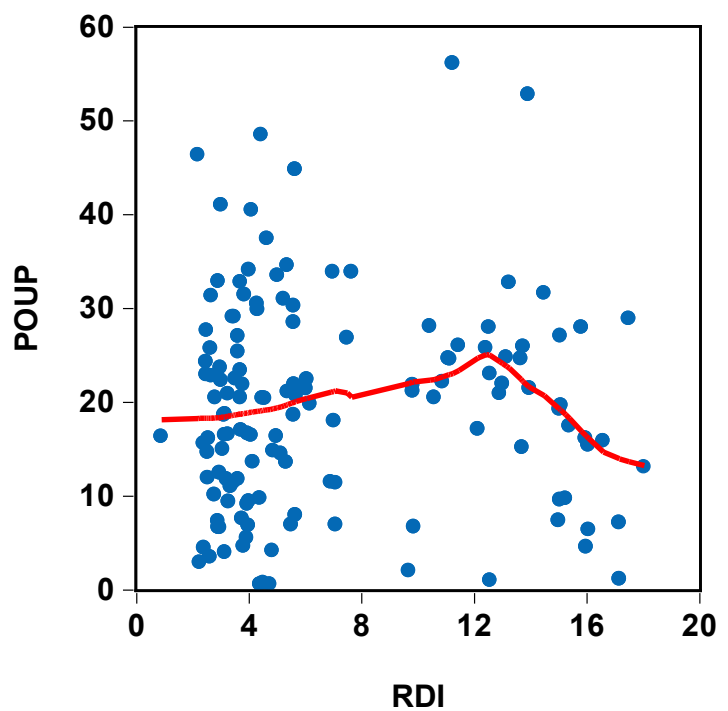

Fonte: World Development Indicator (WDI) e United Nations, Department of Economic and Social Affairs: Population Division, Population Estimates and Projections Section

Desta forma países cuja transição demográfica é tal que sua razão de dependência de idosos for maior que $8 \%$ e esteja crescendo começarão experimentar restrições ao crescimento econômico.

Enquanto que no Brasil os estudos envolvendo transição demográfica tem destacado os efeitos positivos da passagem do bônus demográfico ao longo da década de 1970 até a década de 2000, mais recentemente alguns estudos começam apontar para limites que transição demográfica começa impor na economia. Os resultados obtidos pelas estimativas de Kernel corroboram as preocupações apontadas por Arbache (2011), de que o envelhecimento da 
população e mais especificamente a queda da taxa de crescimento da força de trabalho tende reduzir a competitividade da economia por meio do mercado de trabalho. Adicionalmente os Kernels mostram o país

Uma discussão sempre presente quando se discute a capacidade de crescimento da economia brasileira é alegada falta de poupança para financiar os investimentos e promover o crescimento. De fato, a baixa taxa de poupança dos últimos 20 anos é um problema macroeconômico que o Brasil não conseguiu resolver, de forma que tem colhido como resultado a menor taxa de crescimento entre os BRICS e alguns outros países da América Latina. O problema da poupança tende a se agravar doravante, e se depender da dinâmica demográfica dos próximos 10 a 20 anos, o esforço de aumento da taxa de poupança além de romper com as restrições macroeconômicas já existem deverão também neutralizar os efeitos demográficos que se tornarão progressivamente mais forte no futuro não distante.

Tendo em vista que a mudança demográfica é uma variável fora do controle de qualquer política macroeconômica, o reencontro do país com o crescimento econômico no futuro dependerá de programas e políticas educacionais mais agressivas do que as implementadas até o momento, como forma de preparar as bases para uma economia baseada em inovação, mais do que uma economia baseada acumulação de capital pura e simples.

\section{Referências}

Alves, J. E. D.; Vasconcelos, D. de S.; Carvalho, A. A. de. (2010). "Estrutura etária, bônus demográfico e população economicamente ativa no Brasil: cenários de longo prazo e suas implicações para o mercado de trabalho", Textos para Discussão CEPAL - IPEA n. 10, Brasília.

Arbache, Jorge; (2011). Transformação demográfica e competitividade internacional da economia brasileira, Revista do BNDES, Vol (36), p. 365-392

Azzoni CR, Menezes-Filho NA, Menezes TA; (2005) Opening the convergence black box: measurement problems and demographic aspects. In: Kanbur R, Venables A (eds) Spatial Inequality and Development, UNU-Wider Studies in Development Economics, Oxford Academic Press, pp. 61-74

Berquó, E.; Garcia, S.; Lago, T. (Coord.). Pesquisa Nacional de Demografia e Saúde da Criança e da Mulher: PNDS, 2006. São Paulo: Cebrap, 2008. (Relatório final). Disponível em: <http://bvsms.saude. gov.br/bvs/pnds/img/relatorio_final_pnds2006.pdf>. Acesso em: 20/02/2013.

Birdsall, Nancy; Kelley, Allen C. and Sinding, Steven (eds); 2003. Population Matters: Demographic Change, Economic Growth, and Poverty in the Developing World. Oxford-USA: Oxford University Press.

Bloom, DE.; Canning, D.; Mallaney, P.N.; (1999). Demographic Change and Economic Growth in Asia, Center for International Development (CID), Working Paper $\mathrm{n}^{\circ} 15$, Harvard University.

Cass, D. (1965). Optimum growth in an aggregative model of capital acumulation. Review of Economic Studies, 32:233-240. 
Cutler, David; Poterba, James; Sheiner, Louise; Summers, Larry and Akerlof, George; (1990). An Aging Society: Opportunity or Challenge, Brookings Papers on Economic Activity 1990: p. $1-73$.

Dyson, Tim; (2010). Populaton and Development: The Domographic Transiction, EUA: Zed Books.

Domar, E. D. (1946). Capital expansion, rate of growth, and employment. Econometrica, 14(2):137-147.

Eggleton, Karen and Tujiaporkar, Shripad (eds); (2011).Aging Asia: The Economic and Social Implications of Rapid Demographic Change in China, Japan, and South Korea, The Walter H. Shorenshein Asia-Pacific Research Center

Fukuda S, Morozumi R (2004) Economic growth under the demographic transition: a theory and some international evidence. In: Paolo Onofri (ed) The economics of an ageing population, Edward Elgar, Cheltenham/UK, pp. 3-34

Galor, Oded; (2011). Unified Growth Theory, New Jersey: Princeton University Press.

Harrod, R. F. (1939). An essay in dinamic economics. The Economic Journal, 49, No. 193:14-33.

Higgins, M. and Willianson, J.G.; (1996). Asian demography and foreign capital dependence, NBER Working Paper, no 5560, Cambridge-MA.

Horioka, C. Y.; (1991). The determinants of Japan's saving rate: the impacto of the age structure of the population and other factors, Economic Studies Quarterly, vol (42), p. 237253.

Koopmans, T. C. (1965). On the Concept of Optimal Economic Growth. Elsevier, Amsterdan: North Holland. in The Econometric Approach of Development Planning.

Lee, Ronald; (2003). The Demographic Transition: Three Centuries of Fundamental Change, Journal of Economic Perspectives $\operatorname{Vol}(17)$.

Mason, A.; (2005). Demographic transition and demographic dividends in developed and developing countries. Ciudad del Mexico: United Nations Expert Group Meeting on Social and Economic Implications of Changing Population Age Structure.

Neyer, Gerda et all (eds); (2013). The Demography of Europe. Springer

Prettner K, Prskawetz A.; (2010). Demographic change in models of endogenous economic growth: a survey, VID Working Paper 08/2010, Vienna Institute of Demography

Solow, R. M. (1956). A contribution to the theory of growth. Quarterly Journal of Economics, 70:65-94.

Stampe, Marianne Z.; Porsse, Alexandre A; Portugal, Marcelo S., Almeida, Eduardo S. de, (2012). Demographic Change and Regional Economic Growth in Brazil, mimeo.

Shoven, John B.; (2011). Demography and the Economy, National Bureau of Economic Research Conference Report, EUA: University of Chicago Press.

Swan, T. W. (1956). Economic growth and capital accumulation. The Economic Record, 32:334361.

The National Academy of Sciences, (2012). Aging and the Macroeconomy: Long-Term Implications of an Older Population. Board on Mathematical Sciences and Their Applications - Division on Engineering and Physical Sciences Committee on Population · Division of Behavioral and Social Sciences and Education.

Weber, Lars; (2010). Demograpfhic Change and Economic Growth: Simulations and Growth Models, 